\title{
Detecting Difficult Minor Elements in Particle Samples by SEM-EDS
}

\author{
Abigail P. Lindstrom ${ }^{1}$ and Nicholas WM Ritchie ${ }^{1}$ \\ 1. Materials Measurement Science Division, National Institute of Standards and Technology, \\ Gaithersburg, MD
}

A question that often arises when reviewing particle analysis data is whether we would have seen an element if it were present in a particle. Detection is particularly challenging because particle analysis is typically performed using short acquisition times and the sample preparation is far from the optimal flat, polished bulk samples.

One empirical way to develop confidence in detection limits is to run samples consisting of challenging materials. One class of challenging materials includes the series of NIST glasses containing varying amounts of $\mathrm{Ti}$ in a $\mathrm{Ba}$, Si oxide matrix. Barium and titanium have a particularly challenging overlap in the characteristic line families that are typically used to quantify these elements, the Ti K and Ba L line families. The NIST glasses contain between $1.7 \%$ and $16.2 \%$ Ti by mass, $43.0 \%$ Ba by mass with the remaining mass fraction consisting of $\mathrm{Si}$ and $\mathrm{O}$.

Using the automated particle analysis application on an ASPEX (now a division of FEI) Personal SEM ${ }^{1}$ we collected spectra from approximately 1,000 particles of each glass. The particles were prepared from bulk shards in a cryomill and were typically less than $5 \mu \mathrm{m}$ in diameter. They were prepared for analysis on a standard carbon sticky tab (GSR pin.) The analysis was done at $25 \mathrm{keV}$ with a $20 \mathrm{~s}$ live time dwell on each particle. This live time is much larger than is typically used. However, it is possible to simulate shorter live times by deconstructing the spectrum and randomly assigning acquisition times to each $\mathrm{x}$-ray. The spectrum can then be partitioned into spectra representing arbitrary live times less than 20 seconds.

The particle data was processed using NIST Graf, a quantitative x-ray microanalysis program designed specifically for particle data sets. NIST Graf uses the same quantitative algorithms as NIST DTSA-II. Rather than misapplying ZAF or $\varphi(\rho z)$ corrections to particle data, Graf reports normalized k-ratios (often with C and $\mathrm{O}$ k-ratios removed). These normalized k-ratios can be related to composition but are more useful for clustering particle classes. Removing $\mathrm{O}$ which tends to vary substantially has the advantage of reducing the variance in the other elements thereby facilitating manual and automated clustering.

In particle analysis, there is always a trade-off between the length of an analysis and the desire to analyze many particles. Long analysis times may help resolve interferences, but also reduce the total population that can be sampled. Short analysis times can generate more statistically significant particle populations. Fortunately, these studies have demonstrated that long acquisition times are not necessary to identify low concentrations of Ti obscured by Ba with high confidence $(\mathrm{P}>95 \%)$. See Table 1 for the results of the analysis of two such glasses K2466 and K2496. K2466 is $43.0 \%$ Ba, 4.8 \% Ti, $20.6 \%$ Si and $31.6 \% \mathrm{O}$. K2496 is $43.0 \% \mathrm{Ba}, 1.8 \% \mathrm{Ti}, 22.9 \% \mathrm{Si}$ and $32.3 \% \mathrm{O}$ mass fraction. The table shows the mean and standard deviation of the normalized k-ratio for $\mathrm{Ba}, \mathrm{Si}$ and Ti. Even for K2496

\footnotetext{
${ }^{1}$ Certain products, either public domain or commercial, are identified in this paper. Identification of such products does not imply recommendation or endorsement by NIST, nor does it imply that the identified product is the best available.
} 
with $1.8 \% \mathrm{Ti}$, the standard deviation exceeds the mean for acquisitions of 2 seconds or longer. Figure 1 shows the data from K2496 in a ternary format which makes the relationship between live-time and variance evident.

Table 1. The k-ratios from the quantification of the full and sub-sampled spectra from the two particle samples. The values represent the mean and standard deviation of approximately one-thousand individual particle spectra for each material. The mean is relatively independent of live time. The standard deviation includes a fixed component from particle geometry effects and a variable component from the count statistical variance which increases as live time decreases.

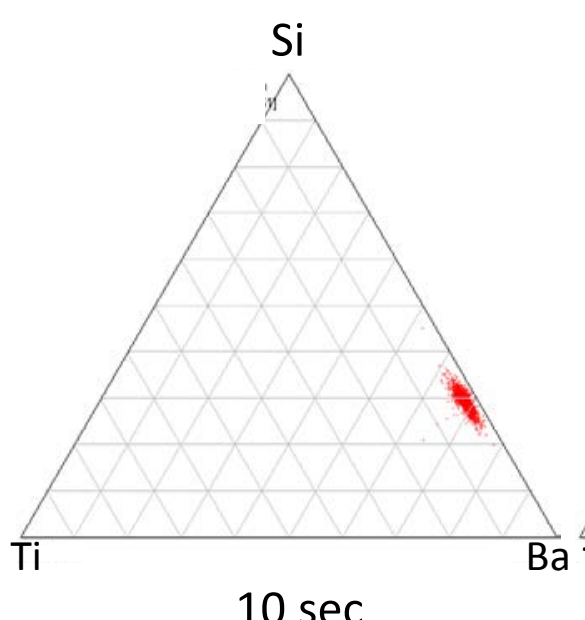

$10 \mathrm{sec}$

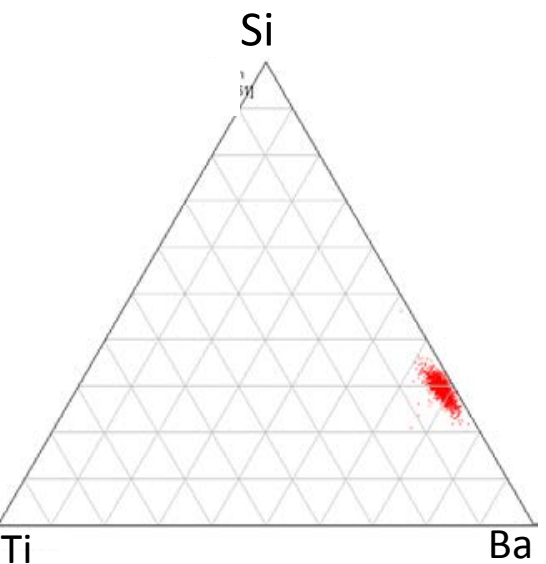

$5 \mathrm{sec}$

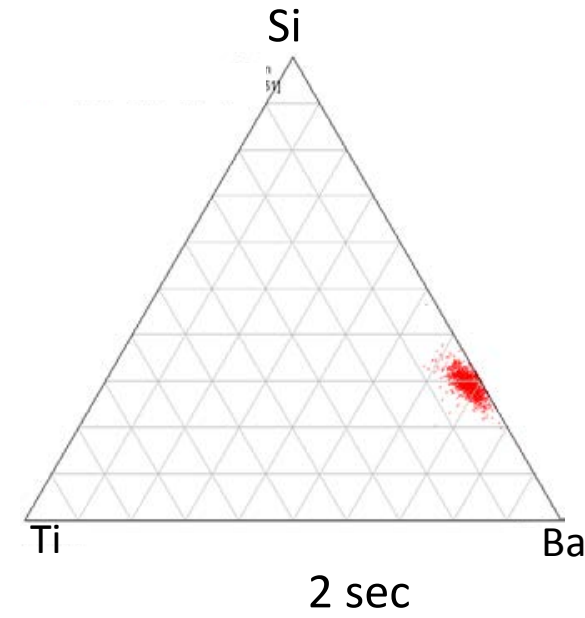

Figure 1. Ba, Si, Ti Ternary diagrams for the analysis of the K-2496 glass at different sub-sampling times. Only the small fraction of particles on the $\mathrm{Si}-\mathrm{Ba}$ axis would register as containing no Ti. 